\title{
Fatigue in laser shock peened open-hole thin aluminium specimens
}

\author{
Goran Ivetic Ivan Meneghin Enrico Troiani Gianluca Molinari José Ocaña Miguel Morales \\ Juan Porro Agostino Lanciotti Vjola Ristori Claudia Polese Jasper Plaisier Andrea Lausi
}

\begin{abstract}
A B S T R A C T
An experimental study was performed in order to determine the influence of the sequence of operations on the effectiveness of Laser Shock Peening (LSP) treatment in increasing the fatigue performances of open-hole aluminium specimens.

Residual stress measurements, fractographic analysis and FEM analysis were performed, indicating the presence of compressive residual stresses on the surface of the treated specimens and tensile residual stresses in the mid-section along the thickness of the specimens. Negative effects on fatigue lives were encountered on the specimens with the hole already present, while positive effect were observed in specimens in which the hole was drilled after LSP treatment.

These results indicate that LSP can be a good solution for "in production" application, in which open holes are to be drilled after the LSP treatment. The application in which LSP is used "in service" on structures with pre-existing cut-outs, has proven to be impracticable in the investigated configuration.
\end{abstract}

\section{Introduction}

Laser shock peening (LSP) is a relatively recent technique used for the insertion of compressive residual stresses in metallic materials. Since its beginning in the 1960s [1] its benefits have been proven for different engineering materials (steel [2-4], titanium [5-8] and aluminium alloys [9-12]).

LSP uses laser generated shock waves to insert compressive residual stresses in treated specimens. High power density laser beam hits the surface of the specimen to be treated. Locally, the laser creates a high pressure plasma that, contained by a thin layer of water flowing on the specimen surface, generates a pressure weave toward the thickness of the specimen. As a consequence of the plastic deformation of the material induced by the pressure wave, compressive residual stresses are established in the LSP treated specimen. A good overview of the LSP technology, its principles and applications can be found in Refs. [13] and [14].

There are two different approaches to the problem; one uses high energy laser pulses (up to $100 \mathrm{~J}$ ) combined with the protective coating, while other, called laser peening without coating (LPwC) [15] uses lasers with lower energies (order or few Joules), higher overlapping of laser peens and no coating. The latter approach has been used in this work.

The lasers used for LSP treatment are usually Q-switched systems, Nd-glass or Nd-YAG type, with wavelength of $1064 \mathrm{~nm}$ and pulse duration range of 10-100 ns [13]. The power densities used for the process go up to $10 \mathrm{GW} / \mathrm{cm}^{2}$; increasing the power density beyond this level does not bring additional advantages due to dielectric breakdown of confining water that limits the peak pressure of plasma developed by the process [16].

In the case of LPWC, shorter wavelength lasers ( $532 \mathrm{~nm})$ are used as well [15]. In this case, the pulse duration is up to $10 \mathrm{~ns}$ and lasers usually have lower output energies. In order to obtain comparable power densities as in the case of LSP treatment with coating, smaller laser peen spots are used.

However, in the case of treatment of thin sheets made of ductile materials, such as aluminium alloys, particular attention is necessary due to the fact that incorrect process setup can create detrimental effects on the fatigue performances of the treated specimens. In fact, there are relatively few works that deal explicitly with the application of LSP technology on thin aluminium specimens. Yang et al. in Ref. [17] describe the application of LSP on thin specimens in $\mathrm{Al} 2024-\mathrm{T} 3$ with the presence of fastener holes. In this case, the treatment was performed on both sides simultaneously in order to avoid distortions and beneficial effects on fatigue lives were found. 
Table 1

The settings of the used laser.

\begin{tabular}{|c|c|c|c|c|c|}
\hline Laser type & Wavelength [nm] & Output energy [J] & Pulse duration [ns] & Peen size [mm] & Laser frequency $[\mathrm{Hz}]$ \\
\hline Nd-YAG & 1064 & $2.8(10 \%$ loss $)$ & 9 & 1.5 & 10 \\
\hline
\end{tabular}

Similar investigation is reported in Ref. [18], in which the authors describe the use of LSP for suppressing the fatigue crack growth of $\mathrm{Al}$ alloys with various preexisting notch configurations (fastener hole, an elliptical hole, one and three in-line crack stopholes and single-edge notch).

Toparli and Fitzpatrick in Ref. [19] describe the application of LSP on thin 2024-T3 aluminium specimens, giving the obtained residual stresses for different process setups. In this case, the treatment was performed on one side of the specimen, only. They conclude that a correct setup in the case of the treatment of thin aluminium specimens is crucial. In all of these works cited, the used LSP approach was the one with protective coating, big laser peen spots (several square millimeters) and high energy lasers, while in the work presented here, low energy laser with high peen overlapping and no protective coating was used.

Previous work done by the authors on the subject of the application of LSP treatment on thin, open-hole specimens [20] with LPwC approach has proven that the LSP effect on fatigue life of treated specimens can be detrimental, if the process is not properly optimized. In fact, it was shown that the capability of the LSP to introduce compressive residual stresses around fastener holes in thin-walled structures representative of typical aircraft constructions was not superior to the performance of conventional techniques, such as cold-working. Cold-working inserts locally residual stresses inside the hole, while this is not the case of LSP, in which only the surface of the specimen is compressed; however in the latter case it is possible to treat much wider areas around the hole, influencing potentially not only the crack nucleation, but its propagation, as well.

The encountered reduced performance of LSP can be attributed to different factors, including the fact that the treatment was performed on the specimens with an open-hole already present. This was not an issue in the works [17] and [18] in which laser peens were much bigger than the fastener holes, however in the case reported here relatively small peen size in respect to the dimension of the hole can cause unwanted effects. In fact, it was shown in Ref. [20] that the effect of the presence of the hole introduced unwanted tensile residual stresses at the inner side of the hole, causing the premature fatigue failure of the specimens in the investigated LSP configuration.

Therefore an additional experimental campaign on the specimens in 6082-T6 was defined in order to highlight the importance of the sequence of operations in the LPWC configuration, which are the drilling of the hole and the LSP treatment.

\section{Experimental setup}

The specimens used for the present research were dog-bone specimens, obtained from $3 \mathrm{~mm}$ thick sheet of Aluminium alloy 6082-T6 (Fig. 1).

Dog-bone specimens were prepared using a CNC machine and subsequently LSP treated at the Polytechnic University of Madrid, using the laser with characteristics described in Table 1 . The peen overlap was 625 peens on square centimeter, with circular peens of $1.5 \mathrm{~mm}$ in diameter. The experimental setup is illustrated in Fig. 2.

When thin specimens are laser peened, they are usually fixed rigidly to a backing plate in order to avoid unwanted shock wave reflections on the back side of the specimen. However, this rigid

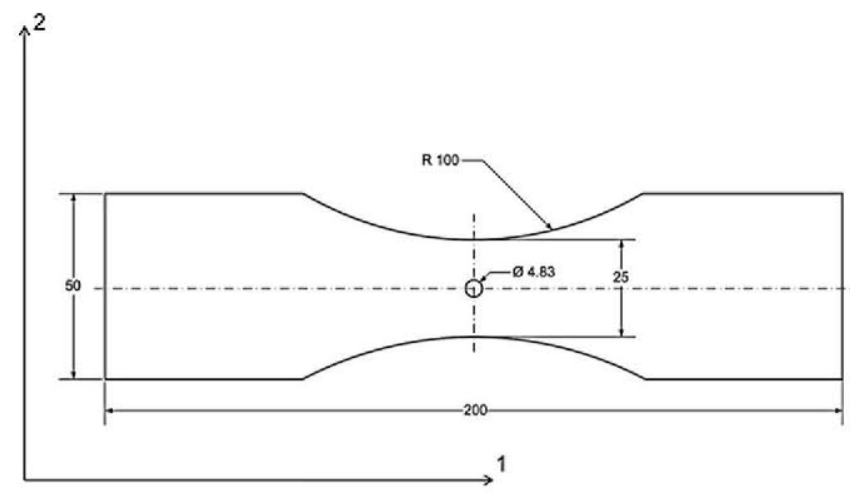

Fig. 1. Geometry of the specimen.

fixing can cause undesirable local variation in residual stresses introduced in the specimen.

Therefore, it was decided to fix the specimen in two points only, allowing it to deform freely, in combination with short impulse times that in fact ensure elastic shock reflection on the back side of the treated specimen.

Residual stresses inserted by the LSP treatment were measured at Elettra Synchrotron facility, MCX Beamline, in Trieste, Italy. The residual stresses were measured at the edge of the open hole, for the two different types of specimens (specimen LSP treated with pre-existing open hole, i.e. hole before, and specimen with the hole drilled on the LSP treated area, i.e. hole after).

Fatigue tests were performed at $R$ ratio of 0.1 for maximum nominal stresses ranging from $120 \mathrm{MPa}$ to $160 \mathrm{MPa}$. Additional tabs were bonded to the clamped sides of the specimens in order to avoid fretting fatigue damage. A total of 15 specimens was tested; five for each case (hole before, hole after, baseline). Even if five specimens are not sufficient for constructing a statistically significant $\mathrm{S}-\mathrm{N}$ curve, the trends obtained from fatigue testing are very clear.

An analysis of fracture surfaces of the two types of specimens ("hole before" and "hole after") was performed at the University of the Witwatersrand, South Africa, using a Scanning electron microscope (SEM) FEI NOVA 600 NanoLab.

\section{Results}

\subsection{Residual stress measurement}

The obtained results relative to synchrotron beam energies of 9 , 12 and $15 \mathrm{keV}$ are given in Table 2 together with the attenuation lengths, i.e. the depths into the material measured along the surface normal, where the intensity of the beam has dropped to $1 / \mathrm{e}$ of its initial value. The actual depth of the measurement is one half of the attenuation length relative to the applied beam energy, i.e.

Table 2

Residual stresses $\left(\sigma_{1}\right)$ at the edge of the open hole.

\begin{tabular}{rlcl}
\hline Energy $[\mathrm{keV}]$ & $\begin{array}{l}\text { Attenuation } \\
\text { length }[\mu \mathrm{m}]\end{array}$ & $\begin{array}{l}\text { Residual stress } \\
\text { hole before [MPa] }\end{array}$ & $\begin{array}{l}\text { Residual stress } \\
\text { hole after [MPa] }\end{array}$ \\
\hline 9 & 109 & -76.30 & -127.09 \\
12 & 256 & -46.22 & -129.54 \\
15 & 497 & -117.68 & -175.81 \\
\hline
\end{tabular}




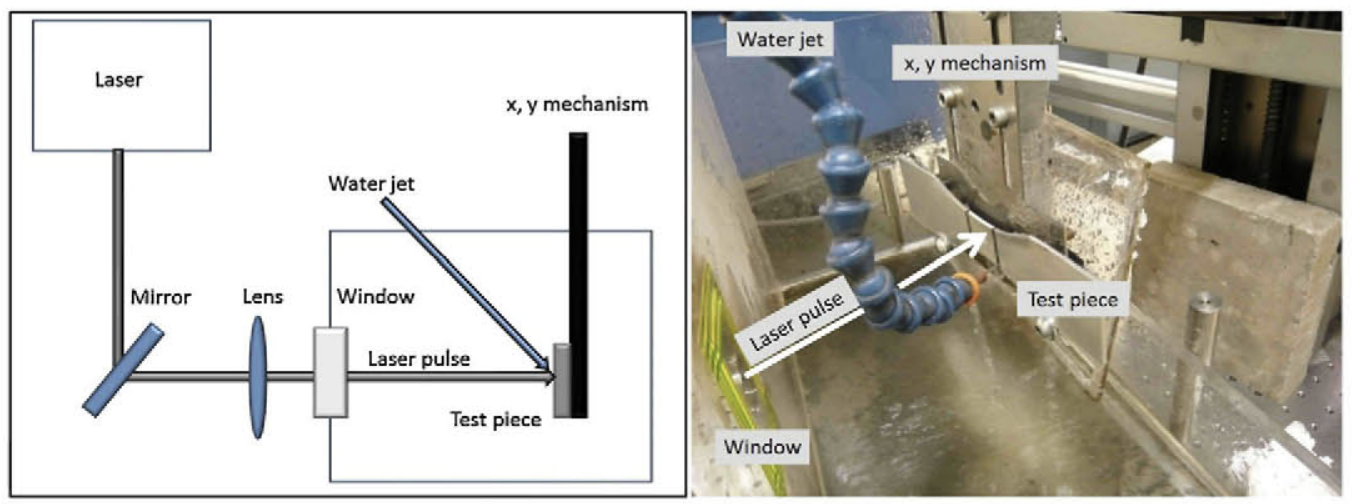

Fig. 2. Experimental setup.

the synchrotron beam needs to enter the material and reflect back to the measuring sensor. A good description of application of synchrotron radiation for residual stress measurement can be found in Ref. [21].

The results are relative to the residual stresses close to the open hole, in the longitudinal direction of the specimen, i.e. the direction of the fatigue loading, Fig. 3. It can be seen that the amount of compressive residual stresses is bigger in the case where the open hole is drilled after the LSP treatment, giving the first indications on the positive effects introduced by drilling the hole only after the LSP treatment has been made.

\subsection{Fatigue testing and fractography}

Table 3 summarizes the results obtained from fatigue testing, while the same results can be seen graphically in Fig. 4 .

First obvious result of this testing campaign is that drilling the hole after the LSP treatment can indeed increase fatigue lives of treated specimen.

In fact, the LSP treatment is effective in increasing the fatigue performances of the thin open-hole specimens when performed before the hole drilling. On the contrary, when the LSP treatment is performed on the specimens with a pre-existing hole, it is detrimental on the fatigue performances.

The fractographic analysis has shown that in fact, both the "hole before" and "hole after" case present crack initiation points in the mid-section of the thickness of the specimen.

Fig. 5 shows the fractographs of the tested specimens for the nominal maximum stresses of 130,140 and $150 \mathrm{MPa}$, respectfully, and the crack initiation point is evidenced with the thick line. It can be seen that for both types of specimens ("hole before" and "hole after") crack initiated beneath the surface of the specimen.

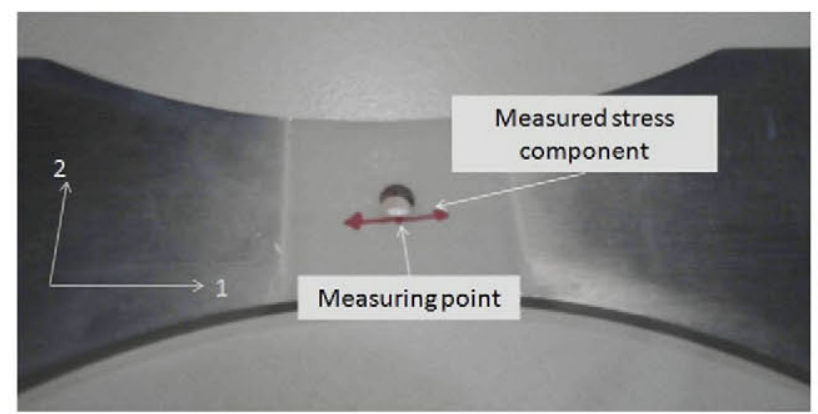

Fig. 3. Examined specimen with the position and the direction of measured residual stresses.

\section{Finite element analysis}

The FEM analysis was performed using a commercial finite element code ABAQUS/Explicit. The aim of this simplified FEM analysis was to obtain the qualitative distribution of residual stresses along the depth of the specimen at the edge of the hole which was difficult to measure experimentally with resources available. The lower compressive residual stresses recorded in the "Hole Before" specimen in comparison with the "Hole After" ones do not justify alone the lower fatigue performances of the "Hole Before" specimens respect to the Baseline.

The approach used for FEM modelling of LSP process defines the behavior of the treated material under shock loading conditions, giving as an input the resulting pressure of the created plasma, rather than modelling the LSP process in its whole, which would increase substantially the complexity of the analysis, and this was the approach used in the present analysis, as well.

The parameters that need to be defined for a simulation of LSP process are the material model and the size and the shape of the surface on which the pressure is applied. The response of the treated material under shock loading, in the context of LSP modelling, is usually described with:

- Hugoniot elastic limit model [22] in which dynamic yield stress and ultimate strength are defined;

- Johnson-Cook material model [23] model defined by parameters that take into account the plastic strain, strain rate and temperature change of the material under shock loading. It is important to emphasize that this material model is purely empirical and that the material constants need to be determined experimentally for each application.

In order to define the necessary input parameters for a FEM analysis, laser power density $\left(\mathrm{GW} / \mathrm{cm}^{2}\right)$ and pulse duration (ns) need to be correlated to the properties of the created shock pulse, i.e. pressure pulse intensity (GPa) and its duration (ns). The results in [16] present experimental correlation between these parameters.

The analysis was composed of a loading step in which the laser peen is applied, and a relaxation step, during which the model returns in an equilibrium state. Both the loading and relaxing steps were performed in ABAQUS/Explicit as suggested in Ref. [24].

The size of the analyzed specimen was $50 \mathrm{~mm} \times 25 \mathrm{~mm} \times 3 \mathrm{~mm}$ with an open hole of $5 \mathrm{~mm}$ in diameter (Fig. 6), simulating the central part of the specimen, only. Encastre boundary condition were defined on two edges of the specimen as illustrated in Fig. 6, simulating the fixing conditions used during the LSP treatment.

The simulated conditions were $2 \mathrm{GW} / \mathrm{cm}^{2}$ and 10 ns pulse duration on a $8 \mathrm{~mm} \times 8 \mathrm{~mm}$ square peen. These process conditions were 
Table 3

Fatigue lives with different operation sequences at $R=0.1$ (BL=baseline).

\begin{tabular}{|c|c|c|c|c|c|}
\hline$\sigma_{\max }(\mathrm{MPa})$ & BL $\left(\right.$ cycles $\left.\times 10^{4}\right)$ & Hole before (cycles $\times 10^{4}$ ) & Hole before/BL (difference) & Hole after (cycles $\times 10^{4}$ ) & Hole after/BL (difference) \\
\hline 120 & 17.54 & 10.46 & 0.59 & 60.04 & 3.42 \\
\hline 130 & 13.32 & 5.68 & 0.42 & 18.16 & 1.36 \\
\hline 140 & 6.58 & 5.41 & 0.82 & 16.08 & 2.44 \\
\hline 150 & 7.87 & 3.87 & 0.49 & 14.25 & 1.80 \\
\hline 160 & 3.99 & 2.62 & 0.65 & 6.63 & 1.65 \\
\hline
\end{tabular}

chosen with the idea of using plausible process setup that could have been used in a hypothetic LSP treatment of thin specimens, maintaining in the analysis the same material model and boundary conditions that would be used in a full analysis that would consider high density of smaller laser peens.

It is important to point out that the performed analysis did not have the aim of simulating actual LSP process used in the experimental part of this work, due to the fact that very high density of laser peens was used which would increase substantially the complexity and the calculation time of the analysis.

In fact, a simplified analysis was performed, simulating one big laser peen only, since the expected result from this analysis was to find the qualitative difference between the "hole before" and "hole after" case.

Two models were considered:

- In the first one the load was applied after the hole was realized.

- In the second one the hole was realized after the LSP treatment simulation, using the model change command available in ABAQUS. This command deactivates the elements that have to be removed, simulating effectively the realization of the hole.

The mesh around the open hole area was denser in respect to the rest of the model, with the element size of about $0.3 \mathrm{~mm}$. The mesh of both the models has been realized using compatible mesh seeds. Total number of elements used is 49,560 CD38R solid 3D elements, 51,920 if the hole is realized after the LSP treatment. Only one laser peen was simulated, with the dimensions $8 \mathrm{~mm} \times 8 \mathrm{~mm}$. This choice reduced to minimum calculation times and allowed to obtain quickly the indications on the differences between residual stress distributions in the two observed cases (drilling of the hole
Table 4

Loading definition.

\begin{tabular}{lllll}
\hline Time & $0 \mathrm{~ns}$ & $5 \mathrm{~ns}$ & $20 \mathrm{~ns}$ & $25 \mathrm{~ns}$ \\
Pressure & $0 \mathrm{GPa}$ & $2 \mathrm{GPa}$ & $2 \mathrm{GPa}$ & $0 \mathrm{GPa}$ \\
\hline
\end{tabular}

Table 5

Johnson-Cook parameters for 6082-T6.

\begin{tabular}{llllll}
\hline$A$ & $B$ & $n$ & $C$ & $m$ & Ref. \\
\hline 428.5 & 327.7 & 1.008 & 0.00747 & 1.31 & [25] \\
\hline
\end{tabular}

before or after the LSP treatment). The laser peens were simulated one shot at a time, one on each side of the specimen.

Loading was defined on a square peen as surface pressure trapezoidal pulse, as reported in Table 4.

The material model used to simulate the behavior of the material under shock conditions was Johnson-Cook model [23], Eq. 1, an empirical model with materials constants available in the literature [25], Table 5.

$\sigma=\left(A+B \epsilon_{e q}^{n}\right)\left[1+C \ln \left(\frac{\dot{\epsilon}}{\dot{\epsilon_{0}}}\right)\right]\left[1-\left(\frac{T-T_{0}}{T_{m}-T_{0}}\right)^{m}\right]$

Residual stresses obtained as a result of this analysis and illustrated in the following figures are $\sigma_{1}$ (the ones in the direction 1 , the direction of fatigue loading), along the depth of the specimen, at the edge of the hole (see directions in Fig. 3 and path in Fig. 6). Fig. 7 illustrates the residual stress distributions obtained for the two observed cases.

Even with an extremely simplified simulation of the LSP process as this one, it can be noted that:

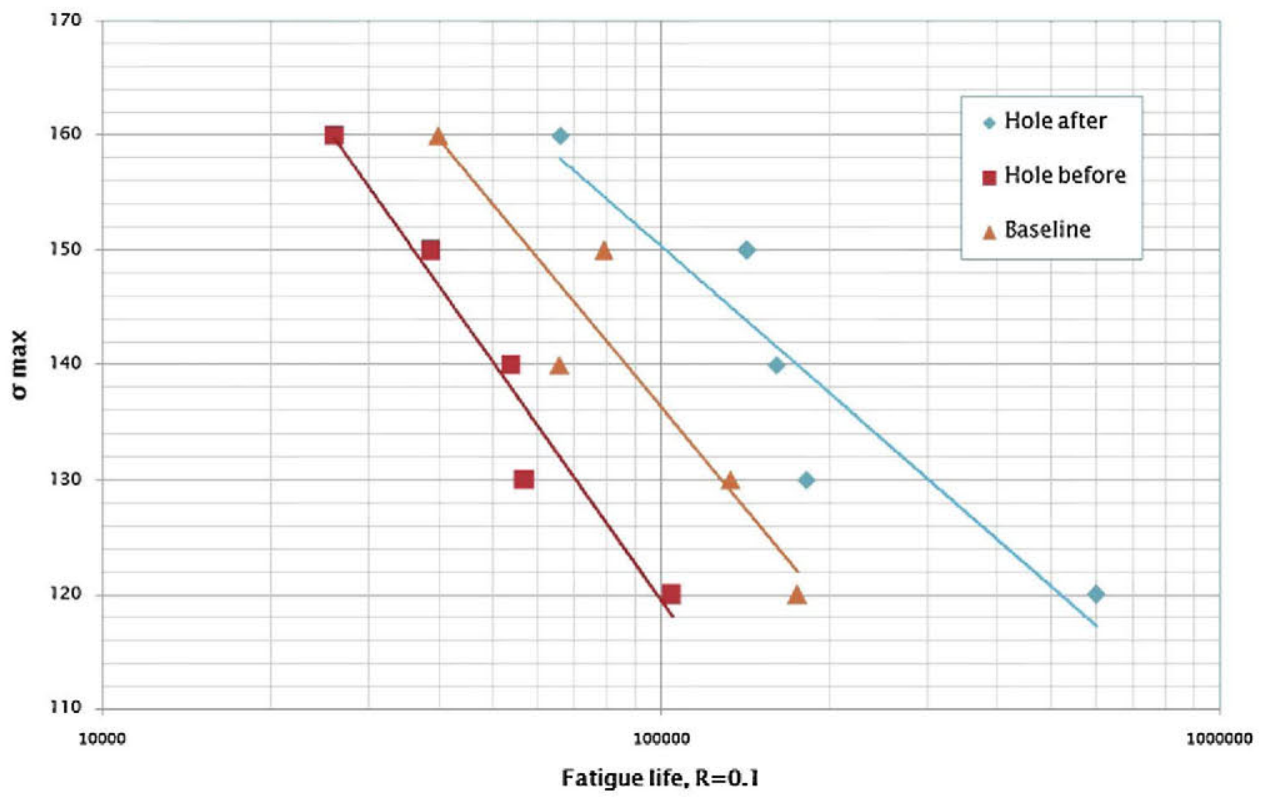

Fig. 4. Effect of the operations sequence: $\sigma_{\max }$ vs fatigue lives of tested specimens. 

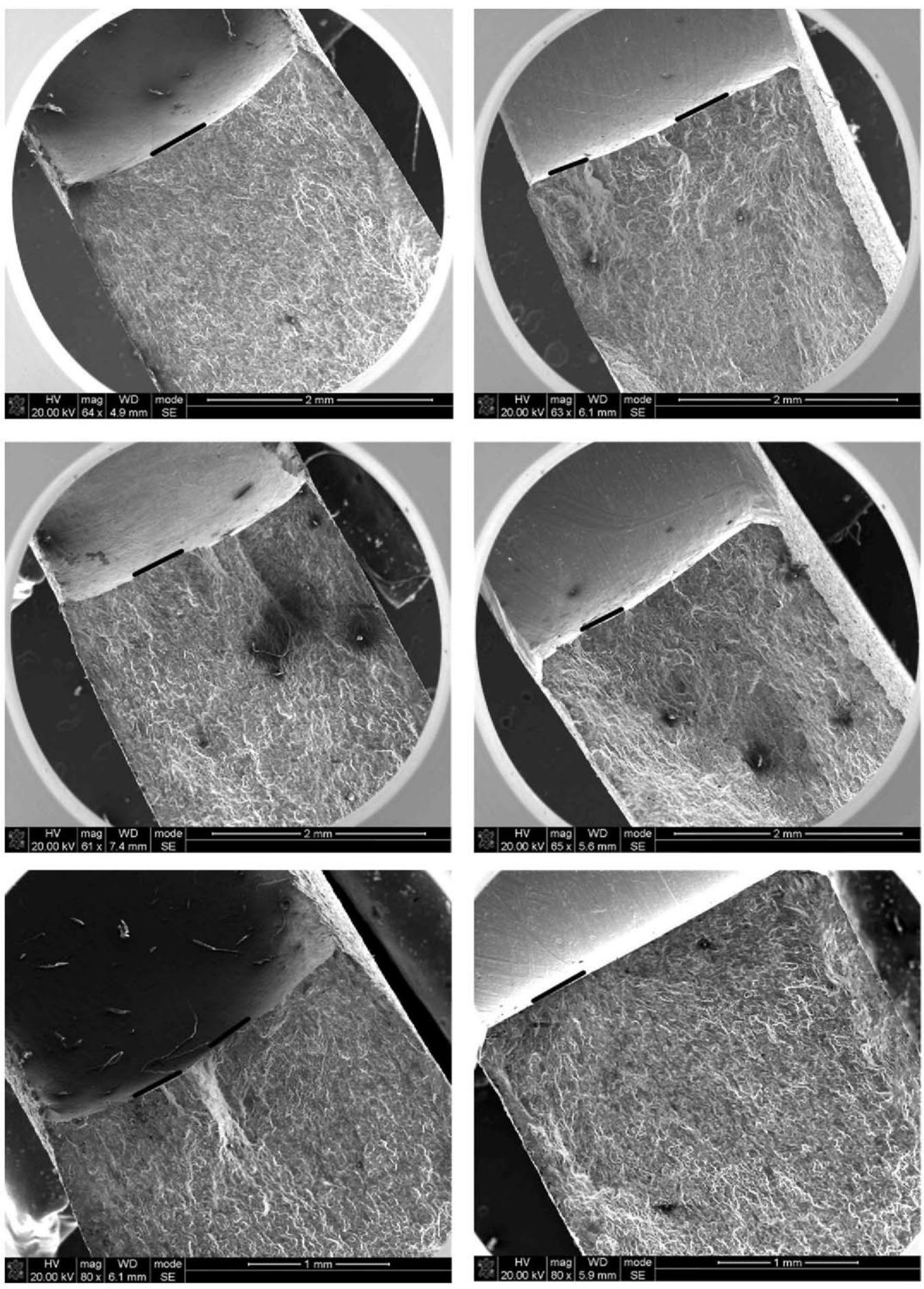

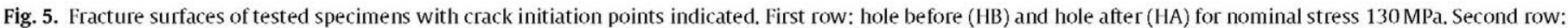
$\mathrm{HB}$ and $\mathrm{HA}$ for nominal stress $140 \mathrm{MPa}$. Third row: $\mathrm{HB}$ and HA for nominal stress $150 \mathrm{MPa}$.

- If the LSP treatment is realized after the hole is drilled, tensile stresses develop inside the hole. This result is in line with the reduction of fatigue life in comparison with the base line specimens. It is also in line with preliminary residual stress measurements done at Elettra Synchrotron and observations from the previous experimental campaign [20], i.e. the nucleation of the cracks began inside the specimen and not on the surface.

- If the hole is realized after the LSP treatment, the residual stress distribution is completely in compression. This result supports the experimental findings of increased fatigue life of the dog-bone specimens.
It is also important to compare the residual stress distributions between the as-peened case (the dog bone specimen without a hole) and the one in which the hole is realized after the LSP treatment. In Fig. 8 the redistribution of residual stresses once the hole is realized is clearly visible.

Another consideration is reserved to the case of simultaneous treatment on the both sides of the specimens, as suggested in the literature [17]. In this case, the FEM analysis was performed by simulating the laser peen on both sides of the specimen simultaneously and the results are given in Figs. 9 and 10. It can be seen that the residual stress profiles are symmetric in respect to the center 


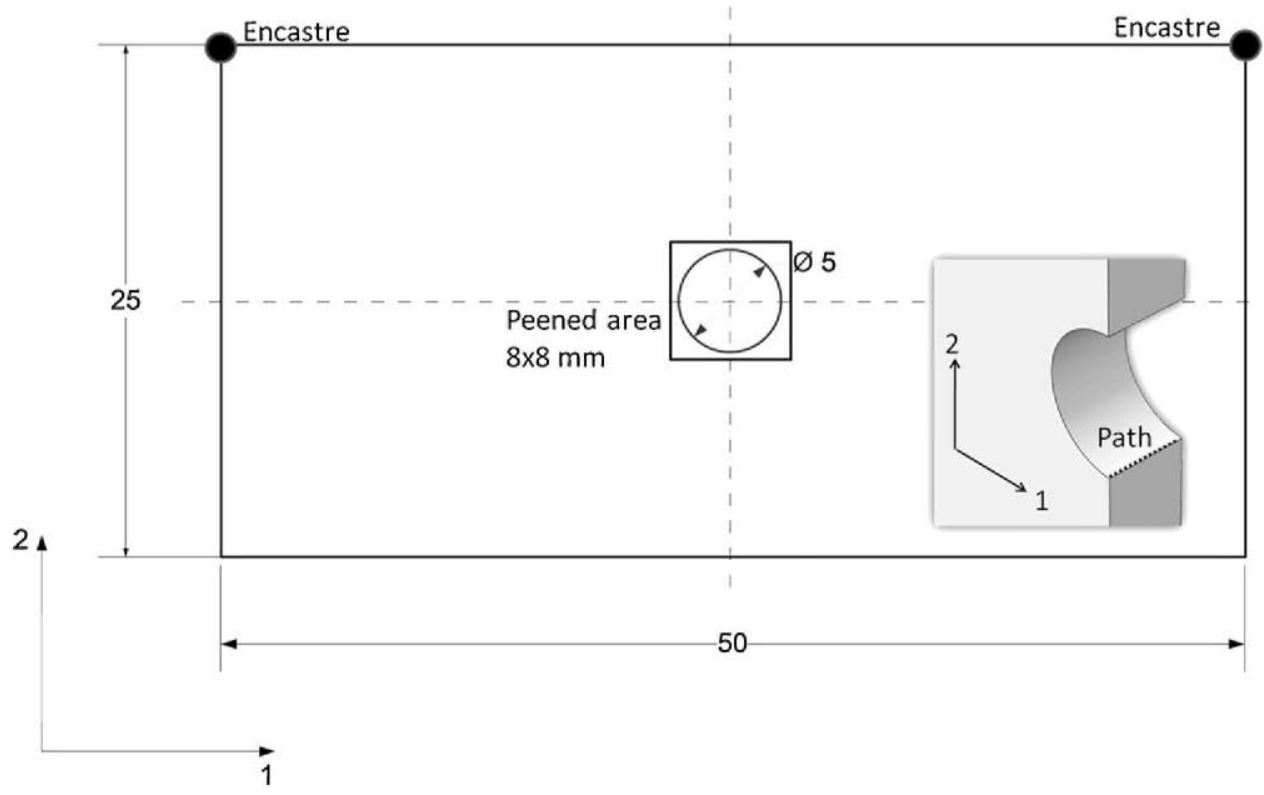

Fig. 6. Geometry considered in the simulation.

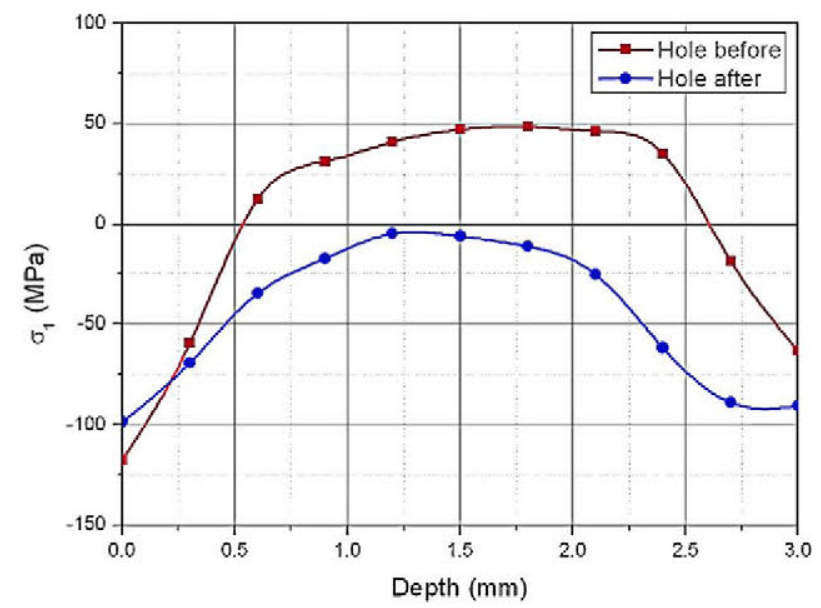

Fig. 7. Residual stresses distribution along the depth of the specimen.

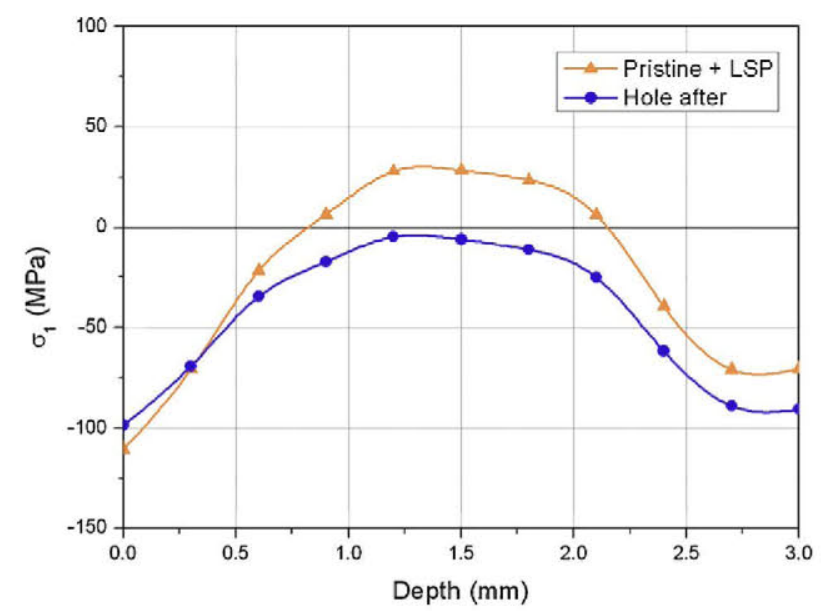

Fig. 8. Residual stresses distribution along the path before and after the hole is realized.

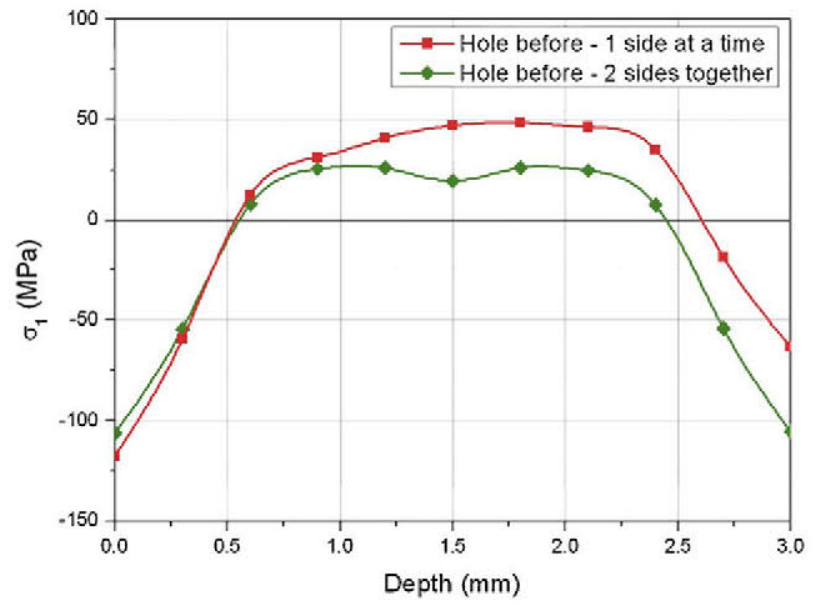

Fig. 9. Residual stresses distributions for the case hole + LSP. The effect of simultaneous peening.

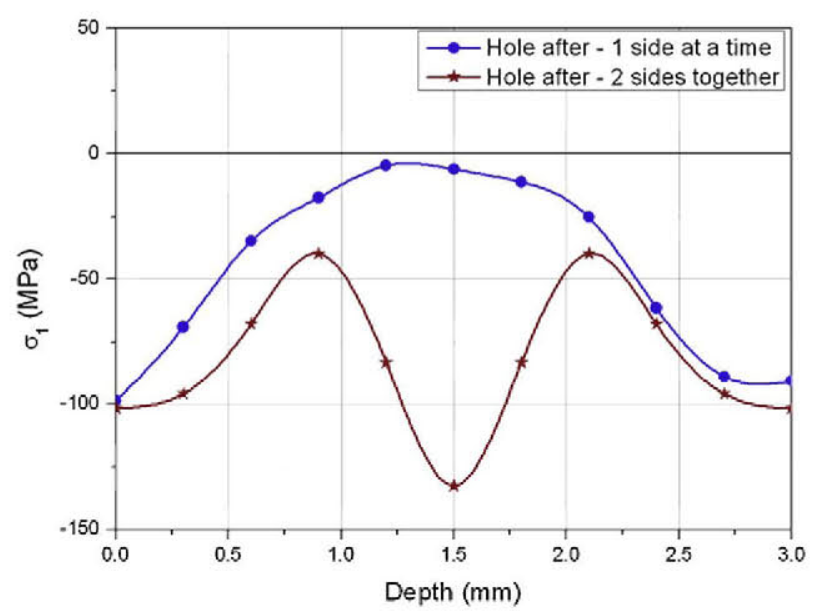

Fig. 10. Residual stresses distributions for the case LSP + hole. The effect of simulta neous peening. 
line and, while in the case of the hole + LSP specimen the results are not showing significant difference, Fig. 9, the case of LSP+hole specimen shows drastically different behavior, Fig. 10.

\section{Discussion}

The practical implications of results obtained in this work is that LSP can be a good solution for "in production" application, in which holes are to be drilled after the LSP treatment. The application in which LSP is used "in service" has proven to be impracticable due to negative effects on fatigue lives that were encountered on the specimens with the hole already present.

Experimental results have indicated compressive residual stresses on the surface of both the types of specimens, and these stresses need to be balanced by tensile stresses beneath the surface. Bearing this in mind, it can be stated that the observed crack initiation points beneath the surface of the specimens are caused by the presence of tensile residual stresses, which are higher in magnitude for the "hole before" case, evidenced by significantly lower fatigue lives.

It is important to point out that the previous research [20] has evidenced that the leaving the "hole before" specimens in aspeened state, polishing them locally inside the hole or polishing them completely, did not show any difference among them in observed fatigue lives. Therefore, the conclusion was that the introduced tensile residual stresses were so high that the effect of the edge of the hole and the roughness of the post-LSP treated surface did not contribute significantly to fatigue results. These residual stresses should be mainly influencing the crack initiation phase, rather than crack propagation phase, due to the progressive relaxation of residual stresses once the crack has formed.

The result of finite elements analysis clearly indicates the importance of the simultaneous peening on both side of the specimen, since treating one side at the time introduces asymmetric residual stress profiles and, furthermore, does not reach the same levels of compressive residual stresses in the mid-section of the specimen, as in two side simultaneous treatment.

\section{Conclusions}

From the results presented in this work, it is possible to draw the following conclusions:

- LSP needs to be optimized for every application used, specially when it comes to low thickness specimens.

- The experiments in the LPwC configuration have shown that the sequence of operations plays a crucial role in increasing the fatigue life of the treated specimens.

- If the hole is realized after the LSP treatment is performed on the specimen, the fatigue life increase is up to three times more than the life of the baseline specimens.

- If the hole is realized before the LSP treatment is performed on the specimen, the fatigue life decrease is about two times less than the life of the baseline specimens.

- Fractographic analysis has indicated that for both types of specimens (hole before and hole after), crack initiated beneath the surface of the specimen.
- Given the difficulty of realizing measurement of the residual stress distribution along the hole, a qualitative approach can be realized numerically using a simplified simulation of the LSP treatment.

- The distributions of residual stresses obtained numerically, have indeed evidenced the differences between the results relative to the realization of the hole before or after the LSP treatment, and their nature corresponds to the experimental findings.

- From the numerical activity it can be seen that realizing the LSP treatment one side at the time is not the best option, performing it simultaneously on both sides should give better results in the terms of compressive residual stresses and so, fatigue life.

\section{Acknowledgement}

The authors wish to acknowledge the support received from the European Science Foundation (ESF) within the activity "Superintense laser-matter interactions".

\section{References}

[1] R.M. White, J. Appl. Phys. 34 (1963) 2123-2124

[2] G. Hammersley, L.A. Hackel, F. Harris, Opt. Lasers Eng. 34 (2000) 327-337.

[3] Y. Sano, M. Obata, T. Kubo, N. Mukai, M. Yoda, K. Masaki, Y. Ochi, Mater. Sci. Eng. A 417 (2006) 334-340.

[4] C. Rubio-Gonzalez, C. Felix-Martinez, G. Gomez-Rosas, J.L. Ocaña, M. Morales, J.A. Porro, Mater. Sci. Eng. A 528 (2011) 914-919.

[5] J.J. Ruschau, R. John, S.R. Thompson, T. Nicholas, Int. J. Fatigue 21 (1999) 199-209.

[6] R.K. Nalla, I. Altenberger, U. Noster, G.Y. Liu, B. Scholtes, R.O. Ritchie, Mater. Sci. Eng. A 355 (2003) 216-230.

[7] C. Cellard, P. Osmond, D. Retraint, E. Rouhaud, S. Remy, A. Viguera-Sancho, in: E.E. Gdoutos (Ed.), Experimental Analysis of Nano and Engineering Materials and Structures, Springer, Netherlands, 2007, pp. 69-70.

[8] S. Spanrad, J. Tong, Mater. Sci. Eng. A 528 (2011) 2128-2136.

[9] C.A. Rodopoulos, J.S. Romero, A.S. Curtis, E.R. de los Rios, P. Peyre, J. Mater. Eng. Perform. 12 (2003) 414-419.

[10] C. Rubio-Gonzalez, J.L. Ocaña, G. Gomez-Rosas, C. Molpeceres, M. Paredes, A. Banderas, J.A. Porro, M. Morales, Mater. Sci. Eng. A 386 (2004) 291-295.

[11] O. Hatamleh, Int. J. Fatigue 31 (2009) 974-988.

[12] U.C. Heckenberger, E. Hombergsmeier, V. Holzinger, W. von Bestenbostel, Int. J. Struct. Integr. 2 (2011) 22-33.

[13] K. Ding, L. Ye, Laser Shock Peening: Performance and Process Simulation, first ed., Woodhead Publishing, Cambridge, 2006.

[14] A. Kruusing, Handbook of Liquids-Assisted Laser Processing, first ed., Elsevier, Oxford, 2008.

[15] Y. Sano, N. Mukai, K. Okazaki, M. Obata, Nucl. Instrum. Methods Phys. Res. B $121(1997) 432-436$

[16] L. Berthe, R. Fabbro, P. Peyre, L. Tollier, E. Bartnicki, J. Appl. Phys. 82 (6) (1997) $2826-2832$.

[17] J.M. Yang, Y.C. Her, N. Han, A.H. Clauer, Mater. Sci. Eng. A 298 (2001) 296-299.

[18] Y. Tan, G. Wu, J.M. Yang, T. Pan, Fatigue Fract. Eng. Mater. Struct. 27 (2004) 649-656.

[19] M.B. Toparli, M.E. Fitzpatrick, Mater. Sci. Forum 681 (2011) 504-509.

[20] G. Ivetic, I. Meneghin, E. Troiani, G. Molinari, A. Lanciotti, V. Ristori, J.L. Ocaña, M. Morales, J.A. Porro, C. Polese, A.M. Venter, ICAF 2011 Structural Integrity: Influence of Efficiency and Green Imperatives 2011, Part 8, pp. 855-866, doi:10.1007/978-94-007-1664-3_66.

[21] P.J. Withers, in: M.E. Fitzpatrick, A. Lodini (Eds.), Analysis of Residual Stress by Diffraction Using Neutron and Synchrotron Radiation, Taylor and Francis, London, 2003, pp. 170-189.

[22] P. Ballard, J. Fournier, R. Fabbro, J. Frelat, J. Phys. IV Coll. 1 (1991) C3-487-C3494.

[23] G.R. Johnson, W.H. Cook, Proc. of 7th Int. Symp. Ball., Hague, The Netherlands, 1983, pp. $541-547$.

[24] P. Peyre, A. Sollier, I. Chaieb, L. Berthe, E. Bartnicki, C. Braham, R. Fabbro, Eur. Phys. J. AP 23 (2003) 83-88.

[25] S.P.F.C. Jaspers, J.H. Dautzenberg, J. Mater. Process. Technol. 122 (2002) $322-330$. 